\title{
ON THE ZERO SET OF A HOLOMORPHIC ONE-FORM ON A COMPACT COMPLEX MANIFOLD
}

\author{
MICHAEL J. SPURR
}

\begin{abstract}
On any compact complex surface $M$, divisors of nonnegative selfintersection which are contained in the zero set (or in the integral set) of a holomorphic 1-form are shown to induce a fibration of $M$ onto a Riemann surface. This result is extended to higher dimensions for $M$ projective. Applications to zero sets of holomorphic 1-forms on surfaces are given.
\end{abstract}

Introduction. The zero set of a vector field on a compact manifold $M$ has for some time been known to convey structural information about $M$. In the compact holomorphic category, zero sets of holomorphic vector fields provide extensions of the Gauss-Bonnet theorem, and, for $M$ Kähler, the dimension of the zero set of a holomorphic vector field relates to cohomological vanishing (via the CarrellLieberman theorem [2]).

In the dual case, the zero set (and more generally the integral set) of a holomorphic 1-form can convey structural information for a compact complex manifold $M$. In Theorem 1, we show that structural information is obtained for any compact surface which carries a holomorphic 1-form pulling back to zero on a divisor $D$ of nonnegative self-intersection. In Theorem 2, we extend this result to the case $M$ is higher dimensional and projective.

We state

THEOREM 1. Let $S$ be any compact complex surface having a holomorphic 1form $\phi$. If $D$ is any divisor on $S$ satisfying $D \cdot D \geq 0$ and $\phi$ pulls back to zero on $D$, then there exists a holomorphic map $f: S \rightarrow R$ onto a compact Riemann surface satisfying

(1) $\phi=f^{*}\left(\phi_{R}\right)$ for some $\phi_{R} \in H^{0}\left(R, \Omega_{R}^{1}\right)$.

(2) $f$ has connected fibers.

(3) Each component of $D$ is setwise contained in a fiber of $f$.

(4) Each connected component of $D$ is a rational multiple of the natural divisor associated to the fiber of $f$ containing it, and so $D \cdot D=0$.

Notationally, $D \cdot D$ is the intersection pairing of $D$ with itself. $\Omega_{M}^{1}$ denotes the sheaf of germs of holomorphic 1-forms on the manifold $M$. A holomorphic 1-form $\phi$ is said to pull back to zero on a divisor $D=\sum_{j=1}^{k} n_{j} D_{j}$ if and only if $i_{j}^{*} \phi=0$ on $D_{j}$ for each $j$, where $i_{j}: D_{j} \rightarrow S$ is inclusion. We remark that if $\phi$ vanishes on $D$ then it necessarily pulls back to zero on $D$, but the converse need not hold.

Received by the editors July 8, 1986 and, in revised form, May 20, 1987.

1980 Mathematics Subject Classification (1985 Revision). Primary 14J25, 14J40; Secondary 14C20, 32J15, 32L99.

Partially supported by NSF Grant DMS 8405207 . 
Theorem 1 and its converse characterize the existence of a holomorphic map $f: S \rightarrow R$ from a compact complex surface onto a compact Riemann surface of genus $g(R) \geq 1$. The converse of Theorem 1 is easily seen to hold: if $f: S \rightarrow R$ is holomorphic onto a compact Riemann surface of genus $g(R) \geq 1$, and if $D$ is the divisor associated to any fiber of $f$, then $D \cdot D=0$ and $f^{*}\left(\phi_{R}\right)$ pulls back to zero on $D$ for any $\phi_{R} \in H^{0}\left(R, \Omega_{R}^{1}\right)$. Note, therefore, that the condition that $D \cdot D \geq 0$ in the theorem is a natural one.

$\S \S 1$ and 2 give the proof of Theorem 1 in the Kähler and non-Kähler cases. $\S 3$ extends Theorem 1 to the higher dimensional, projective case. $\S 4$ gives some remarks and two applications in the surface setting. We mention these here. If a curve $C$ in a compact surface $S$ is contained in the zero set of a holomorphic 1-form then its genus $g(C)$ is bounded above in terms of Chern numbers of $S$. As a partial converse, if $C$ is of sufficiently low genus and has nonnegative self-intersection then $C$ has zero self-intersection and must be contained in the zero set of a holomorphic 1 -form on $S$.

1. Proof of Theorem 1: The Kähler case. In this section we utilize an idea of A. J. Sommese to use the Albanese mapping and the characterization of an exceptional set in $S$ in terms of the negative definiteness of its self-intersection matrix, in showing the Kähler case. We remark that the algebraic case has been shown by C. P. Ramanujam [6] and by F. Catanese [3, p. 510].

Assume that $S$ is Kähler; for the moment it is assumed that $D=\sum_{j=1}^{k} n_{j} D_{j}$ is connected. Without any loss of generality, we also assume $S$ is free of exceptional curves of the first kind.

Let $\alpha_{S}: S \rightarrow A(S)$ be the Albanese mapping of $S$ into its Albanese torus $A(S)$. The method is to produce a subtorus $T_{1}$ of $A(S)$ with $\alpha_{S}\left(\bigcup_{j=1}^{k} D_{j}\right) \subset T_{1}$, but with $\alpha_{S}(S) \not \subset T_{1}$. Defining $T:=A(S) / T_{1}$ as the quotient torus and $Q: A(S) \rightarrow T$ as the quotient map, it will be shown that $Q \circ \alpha_{S}(S)$ in $T$ is one-dimensional. Taking the Stein factorization of $Q \circ \alpha_{S}$

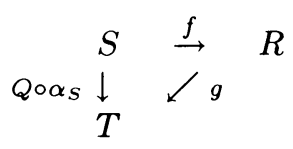

it will be shown that $f: S \rightarrow R$ is the holomorphic mapping in Theorem 1. It will then be shown that $g: R \rightarrow T$ is the Albanese mapping of $R$ into its Albanese torus $A(R)=T$. Hence $Q \circ \alpha_{s}(S)$ is biholomorphic to $R$ and $f$ can be taken to be $Q \circ \alpha_{S}$.

Notationally, for $D=\sum_{j=1}^{k} n_{j} D_{j}$, let $F_{j}: N_{j} \rightarrow D_{j}$ be the normalization of $D_{j}$ and let $i_{j}: D_{j} \rightarrow S$ be inclusion. Choose base points $p_{j} \in N_{j}$ to obtain the Albanese mappings of $N_{j}, \alpha_{j}: N_{j} \rightarrow A\left(N_{j}\right)$ for each $j$. Let $p=i_{1} \circ F_{1}\left(p_{1}\right)$ be the base point in $S$ for $\alpha_{S}$. By the universal property of the Albanese mapping, there are Lie group homomorphisms $q_{j}: A\left(N_{j}\right) \rightarrow A(S)$ and translations $t_{j} \in A(S)$ with $\left(q_{j} \circ \alpha_{j}\right)+t_{j}=\alpha_{S} \circ\left(i_{j} \circ F_{j}\right)$. It is easily seen that $t_{j} \in \operatorname{span}_{j=1}^{k}\left\langle q_{j}\left(A\left(N_{j}\right)\right)\right\rangle$.

Select a basis $\left\{\phi_{1}, \ldots, \phi_{q}\right\}$ for $H^{0}\left(S, \Omega_{S}^{1}\right)$ where $\phi_{q}=\phi$ is the 1 -form pulling back to zero on $D$. Choosing a basis $\left\{\gamma_{1}, \ldots, \gamma_{2 q}\right\}$ for the free part of $H_{1}(S, \mathbf{Z})$ yields a lattice $\Lambda$ in $H^{0}\left(S, \Omega_{S}^{1}\right)^{*}$ generated by $\lambda_{j}, j=1, \ldots, 2 q$, where $\lambda_{j}\left(\phi_{i}\right)=\int_{\gamma_{j}} \phi_{i}$ for $i=1, \ldots, q$. The assumption that $S$ is Kähler implies that $\Lambda$ is a closed 
lattice of rank $2 q$ over $\mathbf{Z}$ in $H^{0}\left(S, \Omega_{S}^{1}\right)$. Then $A(S)=H^{0}\left(S, \Omega_{S}^{1}\right)^{*} \bmod \Lambda$ and $\alpha_{S}(x)=\sum_{i=1}^{q}\left(\int_{p}^{x} \phi_{i}\right) \phi_{i}^{*} \bmod \Lambda$ where $\left\{\phi_{i}^{*}\right\}_{i=1}^{q}$ is the dual basis to $\left\{\phi_{i}\right\}_{i=1}^{q}$.

Recall that $\left(i_{j} \circ F_{j}\right)^{*}: H^{0}\left(S, \Omega_{S}^{1}\right) \rightarrow H^{0}\left(N_{j}, \Omega_{N_{j}}^{1}\right)$ induces

$$
\left(i_{j} \circ F_{j}\right)^{* t}: H^{0}\left(N_{j}, \Omega_{N_{j}}^{1}\right)^{*} \rightarrow H^{0}\left(S, \Omega_{S}^{1}\right)^{*}
$$

and this in turn induces the Lie group homomorphism $q_{j}: A\left(N_{j}\right) \rightarrow A(S)$. Since $F_{j}^{*}\left(i_{j}^{*} \phi_{q}\right)=F_{j}^{*}\left(i_{j}^{*} \phi\right)=F_{j}^{*}(0)=0,\left(i_{j} \circ F_{j}\right)^{* t}\left(H^{0}\left(N_{j}, \Omega_{N_{j}}^{1}\right)\right)^{*} \subseteq\left\{z_{q}=0\right\}$ where $z_{q}$ is the coefficient of $\phi_{q}^{*}$ in $H^{0}\left(S, \Omega_{S}^{1}\right)^{*}$. Hence $q_{j}\left(A\left(N_{j}\right)\right) \subseteq\left\{z_{q}=0\right\} \bmod \Lambda$ and, since $S$ is Kähler $q_{j}\left(A\left(N_{j}\right)\right)$ is a subtorus of $A(S)$ which is not all of $A(S)$. It is an easy consequence that $T_{1}:=\operatorname{span}_{j=1}^{k}\left\langle q_{j}\left(A\left(N_{j}\right)\right)\right\rangle$ is a subtorus contained in $\left\{z_{q}=0\right\}$ $\bmod \Lambda$. Defining $T=A(S) / T_{1}$ with $Q: A(S) \rightarrow T$ the quotient map, we have

$$
\alpha_{S}\left(D_{j}\right)=\alpha_{S}\left(i_{j} \circ F_{j}\left(N_{j}\right)\right)=q_{j}\left(\alpha_{j}\left(N_{j}\right)\right)+t_{j} \subseteq q_{j}\left(A\left(N_{j}\right)\right)+t_{j} \subseteq T_{1}+t_{j} \subseteq T_{1}
$$

for all $j$. Hence $Q \circ \alpha_{S}\left(\bigcup_{j=1}^{k} D_{j}\right) \subseteq Q\left(T_{1}\right)=0$. Since $\alpha_{S}(x)=\sum_{i=1}^{q}\left(\int_{p}^{x} \phi_{i}\right) \phi_{i}^{*}$ $\bmod \Lambda$ and since $\int_{p}^{x} \phi_{q}$ is not identically zero, $\alpha_{S}(S) \not \subset\left\{z_{q}=0\right\} \bmod \Lambda$. So $\alpha_{S}(S) \not \subset$ $T_{1}$ and $Q \circ \alpha_{S}(S) \neq 0$. This fact and the fact that $Q \circ \alpha_{S}(S)$ is a connected analytic set imply $Q \circ \alpha_{S}(S)$ has dimension at least one. It remains to show that

Proposition 1. $Q \circ \alpha_{S}: S \rightarrow T$ has one-dimensional image.

Proof. Assume to the contrary that $Q \circ \alpha_{S}$ has two-dimensional image. We then show that $\bigcup_{j=1}^{k} D_{j}$ is contained in an exceptional set in $S$, contradicting the fact that $D \cdot D \geq 0$.

Recall $Q \circ \alpha_{S}\left(\bigcup_{j=1}^{k} D_{j}\right)=0$. Let $F$ be the connected component of $\left(Q \circ \alpha_{S}\right)^{-1}(0)$ containing $\bigcup_{j=1}^{k} D_{j}$. For

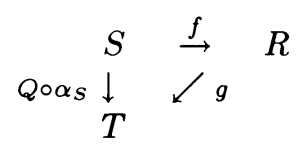

the Stein factorization of $Q \circ \alpha_{S}$, let $p^{\prime}=f(F)$. We remark that, since $S$ and $T$ are normal, $R$ is normal. Let $\mathscr{S}$ be an open neighborhood of $p^{\prime}$ in $R$ such that $f^{-1}(\mathscr{S}) \cap[$ the one-dimensional fibers of $f]=F$ and $f^{-1}(\mathscr{S}) \cap\left[\left(Q \circ \alpha_{S}\right)^{-1}(0)\right]=F$. For $U:=f^{-1}(\mathscr{S})$ we work with the localized Stein factorization

$$
\begin{array}{rlr}
U & \stackrel{f}{\rightarrow} & \mathscr{S} \\
Q \circ \alpha_{S} \underset{T}{\downarrow} & \swarrow_{g} &
\end{array}
$$

$\mathscr{S}$ is irreducible since it is normal. By choosing $\mathscr{S}$ sufficiently small, and by normality and two-dimensionality of $\mathscr{S}, p^{\prime}=f(F)$ is the only possible singularity of $\mathscr{S}$.

Observe next that $F$ is an exceptional set in $U$ via the map $f$. This is done in two steps. First, $F$ is nowhere discrete and satisfies $f:(U \backslash F) \rightarrow \mathscr{S} \backslash p^{\prime}$ is a biholomorphism. The latter follows from Zariski's Main Theorem [10]. The second step is to observe that for all $V$ open $\subset \mathscr{S} f^{*}: \Gamma(V, \mathscr{O}) \rightarrow \Gamma\left(f^{-1}(V), \mathscr{O}\right)$ is an isomorphism. $f^{*}$ clearly injects and it is surjective by normality of $\mathscr{S}$. So $F$ is exceptional in $U$.

$F$ being an exceptional set is characterized by the fact that the matrix $\left(F_{i} \cdot F_{j}\right)$ of the intersection pairings of the irreducible components $F_{i}$ of $F$ is negative definite 
[4]. By assuming $Q \circ \alpha_{S}$ had two-dimensional image, we have concluded $\left(F_{i} \cdot F_{j}\right)$ is negative definite. Recalling that $\bigcup_{j=1}^{k} D_{j} \subset F$ where $D=\sum_{j=1}^{k} n_{j} D_{j}$, let $F_{j}:=D_{j}$ for $j=1, \ldots, k$. Then

$$
\begin{aligned}
0 & \leq D \cdot D=\sum_{i, j}^{k} n_{i} n_{j} D_{i} \cdot D_{j} \\
& =\left(n_{1}, n_{2}, \ldots, n_{k}, 0, \ldots, 0\right)\left(F_{i} \cdot F_{j}\right)\left(n_{1}, n_{2}, \ldots, n_{k}, 0, \ldots, 0\right)^{t} .
\end{aligned}
$$

This contradicts the fact that $\left(F_{i} \cdot F_{j}\right)$ is negative definite, unless $Q \circ \alpha_{S}(S)$ has dimension one, giving the proposition.

To continue the proof of the theorem, observe that $R$ is smooth, as it is normal and one-dimensional. It remains to show that the 1 -form $\phi$ is a pullback via $f$ of some holomorphic 1 -form $\phi_{R}$ on $R$. The mappings $T_{1} \stackrel{i}{\rightarrow} A(S) \stackrel{Q}{\rightarrow} T$ give corresponding mappings of covering spaces:

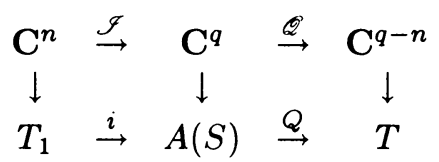

where $q=\operatorname{dim} A(S)$ and $n=\operatorname{dim} T_{1}$. Since $T_{1} \subset\left\{z_{q}=0\right\} \bmod \Lambda, \mathscr{J}\left(\mathbf{C}^{n}\right) \subseteq\left\{z_{q}=\right.$ $0\} \subseteq \mathbf{C}^{q}$. So the $q$ th coordinate $z_{q}$ on $\mathbf{C}^{q}$ induces a coordinate $z$ on $\mathbf{C}^{q-n}$ and thus on $T$. So $d z_{q}=Q^{*}(d z)$ in $H^{0}\left(A(S), \Omega_{A(S)}^{1}\right)$. Now $\alpha_{S}^{*}\left(d z_{q}\right)=\phi_{q}=\phi$ by the choice of basis $\left\{\phi_{1}, \ldots, \phi_{q}=\phi\right\}$ of $H^{0}\left(S, \Omega_{S}^{1}\right)$. Since $Q \circ \alpha_{S}=g \circ f$ in the Stein factorization, $\phi=\alpha_{S}^{*}\left(d z_{q}\right)=\alpha_{S}^{*}\left(Q^{*}(d z)\right)=f^{*}\left(g^{*}(d z)\right)$. Letting $\phi_{R}=g^{*}(d z)$ gives the desired holomorphic form on $R$.

Before completing the proof of the theorem we show that $T$ is the Albanese torus of $R$ and

Proposition 2. $g: R \rightarrow T$ is the Albanese mapping of $R$. Hence $Q \circ \alpha_{S}(S)$ is smooth, biholomorphic to $R$ and $f$ can be chosen to be $Q \circ \alpha_{S}$.

ProOF. One has the commutative diagram

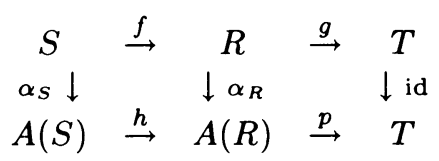

where $h$ is induced by $\alpha_{R} \circ f$ and the universal property of $A(S)$, while $p$ is induced by $g$ and the universal property of $A(R)$. By appropriately selecting the base point of $\alpha_{R}$, we can assume $h$ and $p$ are Lie group homomorphisms. Now $h$ must be surjective, otherwise $h(A(S))$ is the Albanese torus of $R$. Since $p \circ h \circ \alpha_{S}=g \circ f=$ $Q \circ \alpha_{S}$ one has $p \circ h=Q$ on $A(S)$. As $Q$ is surjective, $p$ is. Also $T_{1}=\operatorname{ker} Q=$ $\operatorname{ker}(p \circ h) \subset \operatorname{ker} h$ implies $\operatorname{ker} h=\operatorname{ker}(p \circ h)=T_{1}$. Thus $\operatorname{ker} p=0$ and $p: A(R) \rightarrow T$ is a Lie group biholomorphism. Since the Albanese mapping of a Riemann surface to its Albanese torus is an embedding, $g(R)=Q \circ \alpha_{S}(S)=p \circ \alpha_{R}(R)$ is smooth and biholomorphic to $R$.

To continue the proof of the theorem, we drop the assumption that

$$
D=\sum_{j=1}^{k} n_{j} D_{j}
$$


is connected. If $D$ is not connected, find some connected component $D_{c}$ of $D$ with $D_{c} \cdot D_{c} \geq 0$ and apply the above results to obtain the map $f: S \rightarrow R$. Now each irreducible component of $D$ is contained in a fiber of $f$ (otherwise $f\left(D_{j}\right)=R$ for some $j$ and $i_{j}^{*} \phi=i_{j}^{*} f^{*} \phi_{R}$ is nonzero on $D_{j}$ where $i_{j}: D_{j} \rightarrow S$ is inclusion). In particular each connected component $D_{c}$ of $D$ is contained in a fiber of $f$. A theorem of Zariski [1] gives $D_{c} \cdot D_{c} \leq 0$. Since $D \cdot D \geq 0$ every connected component of $D$ satisfies $D_{c} \cdot D_{c}=0$ and so $D \cdot D=0$. The result of Zariski then gives that, since each connected component $D_{c}$ has self-intersection 0 , each $D_{c}$ is a rational multiple of the divisor associated to the fiber of $f$ containing $D_{c}$. This finishes the theorem in the case $S$ is Kähler.

2. Proof of Theorem 1: The non-Kähler case. To handle the non-Kähler case, note that by Kodaira's classification of compact surfaces and by the fact that $\phi \in H^{0}\left(S, \Omega_{S}^{1}\right) \neq 0, S$ must be elliptic. Let $\pi: S \rightarrow R$ be the elliptic map onto the base curve $R$. There are two cases to handle:

(1) $\phi \in \pi^{*} H^{0}\left(R, \Omega_{R}^{1}\right)$,

(2) $\phi \in H^{0}\left(S, \Omega_{S}^{1}\right) \backslash \pi^{*} H^{0}\left(R, \Omega_{R}^{1}\right)$.

Case (1) is easily dealt with by letting $f=\pi$ and observing that all of the conclusions of the theorem are then satisfied by $f$. In case (2) it will be shown that if an elliptic surface $S$ satisfies the hypotheses of the theorem along with the requirement that the 1-form $\phi \in H^{0}\left(S, \Omega_{S}^{1}\right) \backslash \pi^{*} H^{0}\left(R, \Omega_{R}^{1}\right)$, then $S$ must be Kähler. It follows that case (2) is vacuous.

So assume $S$ is elliptic, satisfies the hypotheses of the theorem, and

$$
\phi \in H^{0}\left(S, \Omega_{S}^{1}\right) \backslash \pi^{*} H^{0}\left(R, \Omega_{R}^{1}\right) .
$$

We rely on the fact that, since $\phi$ is a nonpullback holomorphic 1-form on an elliptic surface, $\phi$ can never vanish on $S$, every fiber of $\pi$ is a smooth torus, and away from multiple fibers $S$ has the structure of a principal torus bundle with constant transition functions [8]. Since $d \phi=0, \operatorname{ker} \phi$ defines an integrable distribution on $S$. Each irreducible component $D_{j}$ of $D$ is a leaf of the resulting foliation. Hence each $D_{j}$ is smooth and intersects no other irreducible component of $D$. Also each irreducible component $D_{j}$ meets every fiber of $\pi$ transversely. This follows since, if $D_{j}$ meets any fiber $F$ of $\pi$ tangentially at $p \in D_{j} \cap F$, then $\phi$ pulls back to zero at $p$ on $F$ and, since $F$ is a torus, $\phi$ pulls back to zero identically on $F$. This implies that $\phi$ pulls back to zero on every fiber of $\pi$ and therefore that $\phi \in \pi^{*} H^{0}\left(R, \Omega_{R}^{1}\right)$ [8], contrary to the assumption of case (2).

Select $D_{1}$ the first irreducible component of $D . \pi: D_{1} \rightarrow R$ is unramified, except at multiple fibers of $\pi$, by transversality of $D_{1}$ to fibers of $\pi$. Let $S$ have multiple fibers $B_{1}, \ldots, B_{J}$ where $B_{i}$ has multiplicity $m_{i}$. Define $S^{\prime}=S \backslash \bigcup_{i=1}^{J} B_{i}$, $D_{1}^{\prime}=D_{1} \cap S^{\prime}$, and $X^{\prime}=\left\{(d, p) \in D_{1}^{\prime} \times S^{\prime} \mid \pi(d)=\pi(p)\right\}$. As mentioned earlier, $S^{\prime}$ is a principal torus bundle over $\pi\left(D_{1}^{\prime}\right)$ having constant transition functions and fiber $F=T$ a torus. This implies $X^{\prime}$ is a principal torus bundle over $D_{1}^{\prime}$, via first factor projection $\pi_{1}$, with constant transition functions and fiber $T$. Second coordinate projection $\pi_{2}: X^{\prime} \rightarrow S^{\prime}$ gives $X^{\prime}$ as an $m$-fold cover of $S^{\prime}$ where $m:=F \cdot D_{1}$, for $F$ a general fiber of $\pi . X^{\prime}$ is seen to be biholomorphic to $D_{1}^{\prime} \times T$ by observing that, if $i_{1}: D_{1} \rightarrow S$ is inclusion and if $\mathscr{I}: D_{1}^{\prime} \rightarrow X^{\prime}$ is the inclusion given by $\mathscr{I}(d)=\left(d, i_{1}(d)\right)$, then $\mathscr{I}\left(D_{1}^{\prime}\right)$ gives a zero section of $X^{\prime}$. 
We next show that the $m$-fold cover $X^{\prime} \simeq D_{1}^{\prime} \times T$ of $S^{\prime}$ can be completed in a natural way to an $m$-fold cover $X$ of $S$, with $X$ biholomorphic to $D_{1} \times T$. This then shows $S$ is algebraic, since its cover $D_{1} \times T$ is. Hence $S$ is Kähler and case (2) is vacuous as claimed, proving Theorem 1 in general.

$X$ is constructed by taking suitable covers $Y_{i}$ of neighborhoods of the multiple fibers $B_{i}$, and patching the $Y_{i}$ into $X^{\prime}$. Examine $S^{\prime}$ near a multiple fiber $B_{i}$. Let $S_{i}^{*}=\pi^{-1}\left(\Delta_{i}\right) \backslash B_{i} \subset S^{\prime}$ where $\Delta_{i}$ is a coordinate disc neighborhood in $R$ centered at $\pi\left(B_{i}\right)$. Let $u_{i}$ be a fixed coordinate function on $\Delta_{i}$ with $u_{i}\left(\pi\left(B_{i}\right)\right)=0$. Define $X_{i}^{*}=\pi_{2}^{-1}\left(S_{i}^{*}\right) \subset X^{\prime}$; explicitly $X_{i}^{*}=\left\{(d, p) \in\left(S_{i}^{*} \cap D_{1}\right) \times S_{i}^{*} \mid \pi(d)=\pi(p)\right\}$. Let $D_{i n}$ be the components of $\pi^{-1}\left(\Delta_{i}\right) \cap D_{1}$ where $n$ ranges from 1 to $m / m_{i}$. (Recall that $m:=F \cdot D_{1}=\left(m_{i} B_{i}\right) \cdot D_{1}$ implies $m_{i} \mid m$ where $m_{i}$ is the multiplicity of $B_{i}$.) Let $D_{i n}^{*}=\left(S_{i}^{*} \cap D_{i n}\right)$. Also let the components of $X_{i}^{*}$ be $X_{i n}^{*}=\left\{(d, p) \in D_{i n}^{*} \times S_{i}^{*} \mid\right.$ $\pi(d)=\pi(p)\}$ and let

$$
Y_{i}=\left\{(p, z) \in \pi^{-1}\left(\Delta_{i}\right) \times \mathscr{O}_{\pi^{-1}\left(\Delta_{i}\right)} \mid z \in \mathscr{O}_{p}, z^{m_{i}}=u_{i} \circ \pi\right\}
$$

where $\mathscr{O}_{\pi^{-1}\left(\Delta_{i}\right)}$ is the sheaf of germs of holomorphic functions on $\pi^{-1}\left(\Delta_{i}\right) . Y_{i}$ has the structure of an $m_{i}$-fold cover of $\pi^{-1}\left(\Delta_{i}\right)$ under first factor projection $\rho_{i}: Y_{i} \rightarrow$ $\pi^{-1}\left(\Delta_{i}\right)$, while each $X_{i n}^{*}$ has the structure of an $m_{i}$-fold cover of $S_{i}^{*} \subset \pi^{-1}\left(\Delta_{i}\right)$ under second factor projection $\pi_{2}$. These covering structures on $Y_{i}$ and $X_{i n}^{*}$ in fact coincide over $S_{i}^{*}$.

To see this, let $u_{i n}$ be a fixed coordinate function on $D_{i n}$ satisfying $\left(u_{i n}\right)^{m_{i}}=u_{i}$. Define $\gamma_{i n}: X_{i n}^{*} \rightarrow Y_{i}$ by $\gamma_{i n}(d, p)=\left(p, z_{d}\right)$ where $z_{d}$ is the function element around $p$ given by (i) $z_{d}^{m_{i}}=u_{i} \circ \pi$ and (ii) $z_{d}(p)=u_{i n}(d)$. $\gamma_{i n}$ gives a biholomorphism of $X_{i n}^{*}$ with $\rho_{i}^{-1}\left(S_{i}^{*}\right) \subset Y_{i}$, as $\gamma_{i n}^{-1}(p, z)=\left(u_{i n}^{-1}(z(p)), p\right)$. Since $\pi_{2} \circ \gamma_{\text {in }}^{-1}=\rho_{i}$, the covering structures on $X_{i n}^{*}$ and $Y_{i}$ agree over $S_{i}^{*}$.

$$
\begin{array}{ccc}
X_{i n}^{*} & \stackrel{\gamma_{i n}}{\longrightarrow} & Y_{i} \\
\pi_{2} \downarrow & & \downarrow \rho_{i} \\
S_{i}^{*} & \stackrel{\text { id }}{\longrightarrow} & S_{i}
\end{array}
$$

Hence for each $n=1, \ldots, m / m_{i}$ a copy of $Y_{i}$ can be patched into $X_{i n}^{*} \subset X^{\prime}$ via the map $\gamma_{i n}^{-1}$. Do this for each multiple fiber $B_{i}$ to obtain the resulting covering space $X$ of $S$, with $\rho: X \rightarrow S$ the covering map induced by $\pi_{2}$ on $X^{\prime}$ and $\rho_{i}$ on $Y_{i}$.

It must be shown next that $X$ is biholomorphic to $D_{1} \times T$. To accomplish this, it is first shown that the inclusion $\mathscr{F}$ of $D_{1}^{\prime}$ into $X^{\prime}$ extends to an inclusion $I$ of $D_{1}$ into $X$. This is done locally by observing that the inclusion of $D_{i n}^{*}$ into $X_{i n}^{*}$ given by $\mathscr{I}(d)=\left(d, i_{1}(d)\right)$ extends to an inclusion of $D_{i n}$ into $Y_{i}$ under $\gamma_{i n}$. Let $u_{i n}$ be the fixed coordinate on $D_{i n}$ as above. Extend $u_{i n}$ to $w_{i n}$ on an open neighborhood of $i_{1}\left(D_{i n}\right)$ in $\pi^{-1}\left(\Delta_{i}\right)$ by requiring $w_{i n}$ to be constant on fibers of $\pi$ and that $w_{\text {in }} \circ i_{1}=u_{\text {in }}$ on $D_{i n}$. Define $I_{i n}: D_{i n} \rightarrow Y_{i}$ by $I_{i n}(d)=\left(i_{1}(d),\left(w_{i n}\right)_{d}\right)$ where $\left(w_{i n}\right)_{d}$ is the germ of $w_{i n}$ at $i_{1}(d)$. Note that for $I_{i n}(d) \in \rho_{i}^{-1}\left(S_{i}^{*}\right) \subset Y_{i}$,

$$
\gamma_{i n}^{-1}\left(I_{i n}(d)\right)=\gamma_{i n}^{-1}\left(i_{1}(d),\left(w_{i n}\right)_{d}\right)=\left(u_{i n}^{-1}\left(w_{i n}\left(i_{1}(d)\right)\right), i_{1}(d)\right)=\left(d, i_{1}(d)\right)=\mathscr{J}(d) \text {. }
$$

So $I_{i n}=\gamma_{i n} \circ \mathscr{I}$ on $D_{i n}^{*}$ for each $i, n$. The $I_{i n}$ together with $\mathscr{I}$ define the inclusion $I: D_{1} \rightarrow X . I\left(D_{1}\right)$ will be the zero section in the trivialization $X \simeq D_{1} \times T$.

The map $\mu_{i n}: Y_{i} \rightarrow D_{i n}$ given by $\mu_{i n}(p, z)=u_{i n}^{-1}(z(p))$ exhibits $Y_{i}$ as a deformation of tori over $D_{i n}$ (there is no multiple fiber over $D_{i n} \cap B_{i}$ ). Since $\mu_{i n}^{-1}$ of any point in $D_{i n}^{*}$ is $T, \mu_{i n}^{-1}\left(D_{i n} \cap B_{i}\right)=T$. Hence $\mu_{i n}$ is trivial over $D_{i n}$. Note that $\mu_{i n}$ 
satisfies $\pi_{1}=\mu_{i n} \circ \gamma_{i n}: X_{i n}^{*} \rightarrow D_{i n}^{*}$ where $\pi_{1}$ is first factor projection on $X_{i n}^{*} \subseteq X^{\prime}$. This follows since

$$
\mu_{i n} \circ \gamma_{i n}(d, p)=\mu_{i n}\left(p, z_{d}\right)=u_{i n}^{-1}\left(z_{d}(p)\right)=u_{i n}^{-1}\left(u_{i n}(d)\right)=d=\pi_{1}(d, p) .
$$

So $\pi_{1}: X^{\prime} \rightarrow D_{1}^{\prime}$ extends to a global projection $\mu: X \rightarrow D_{1}$ with fiber $T$ and no multiple fibers. Recall that $\rho: X \rightarrow S$ was the covering map extending $\pi_{2}: X^{\prime} \rightarrow S^{\prime}$. Now $\rho^{*} \phi$ is a holomorphic 1 -form on $X$ which is not an element of $\mu^{*} H^{0}\left(D_{1}, \Omega_{D_{1}}^{1}\right)$, as $\rho^{*} \phi$ pulls back to zero on $I\left(D_{1}\right) \subset X$. Hence $X$ is a principal torus bundle over $D_{1}$ having constant transition functions and fiber $T[8]$. Letting $I\left(D_{1}\right)$ be the zero section of $X$ over $D_{1}$ gives $X$ is biholomorphic to $D_{1} \times T$ and finishes the proof of the theorem.

3. The higher dimensional projective case. In this section we generalize Theorem 1 to the case in which $M$ is a compact projective algebraic manifold of arbitrary dimension, where $M$ carries a holomorphic 1-form pulling back to zero on a divisor $D$ which satisfies a certain nonnegativity condition.

THEOREM 2. Let $M$ be a compact projective manifold of dimension $n$, with $\omega$ the Kähler form induced by $\mathbf{P}^{N}$. If $M$ carries a holomorphic 1-form $\phi$ which pulls back to zero on a divisor $D=\sum n_{j} D_{j}$ with $\int_{M} c_{1}^{2}(D) \wedge \omega^{n-2} \geq 0$ then there exists a holomorphic map $f: M \rightarrow R$ onto a Riemann surface of nonzero genus with

(1) $\phi=f^{*}\left(\phi_{R}\right)$ for some $\phi_{R} \in H^{0}\left(R, \Omega_{R}^{1}\right)$.

(2) $f$ has connected fibers.

(3) Each component of $D$ is setwise contained in a fiber of $f$.

(4) $\int_{M} c_{1}^{2}(D) \wedge \omega^{n-2}=0$.

ProOF. As in Theorem 1, the inclusions of the $D_{j}$ into $M$ give rise to mappings $i_{j}: N_{j} \rightarrow M$ from the desingularizations $N_{j}$ into $M$. These induce mappings $q_{j}: A\left(N_{j}\right) \rightarrow A(M)$ on the Albanese tori. For $T_{1}=\operatorname{span}_{j=1}^{k}\left\langle q_{j}\left(A\left(N_{j}\right)\right)\right\rangle$ the quotient map $Q: A(M) \rightarrow A(M) / T_{1}$ composed with the Albanese mapping $\alpha_{M}: M \rightarrow A(M)$ gives the desired mapping $f=Q \circ \alpha_{M}: M \rightarrow A(M) / T_{1}$.

The main issue is to show that $f(M)$ is one dimensional, which by using Stein factorization, normality, and Proposition 2, will give that $f(M)$ is smooth. Since $f(M)$ cannot be zero dimensional, we only need show that $\operatorname{dim} f(M) \geq 2$ is prohibited. This is done by slicing $M$ with hyperplane sections.

If $\operatorname{dim} f(M)$ were 2 or more, one could choose a hyperplane $H_{1}$ such that

(a) $M \cap H_{1}$ is smooth.

(b) $H_{1}$ is transverse to $D$ at some point.

(c) $f\left(M \cap H_{1}\right)$ is of maximal dimension.

One could continue slicing with hyperplanes where the requirements in choosing the $j$ th hyperplane $H_{j}$ are

(a) $M \cap H_{1} \cap H_{2} \cap \cdots \cap H_{j}$ is smooth.

(b) $H_{j}$ is transverse to $D \cap H_{1} \cap \cdots \cap H_{j-1}$ at some point.

(c) $f\left(M \cap H_{1} \cap \cdots \cap H_{j}\right)$ is of maximal dimension.

Then $M \cap H_{1} \cap H_{2} \cap \cdots \cap H_{n-2}:=S$ is a surface and $f\left(M \cap H_{1} \cap \cdots \cap H_{n-2}\right)$ has dimension 2. So $D \cap H_{1} \cap \cdots \cap H_{n-2}$ is contained in an exceptional set in $S$ and 
hence the divisor $\widetilde{D}$ on $S$ induced by $D$ satisfies

$$
\begin{aligned}
\widetilde{D} \cdot \widetilde{D} & =\int_{M \cap H_{1} \cap \cdots \cap H_{n-2}} c_{1}^{2}(D) \\
& =\int_{M} c_{1}^{2}(D) \wedge c_{1}^{n-2}(H) \\
& =\int_{M} c_{1}^{2}(D) \wedge \omega^{n-2}<0 .
\end{aligned}
$$

This contradicts the hypothesis that $\int_{M} c_{1}^{2}(D) \wedge \omega^{n-2} \geq 0$ giving that $\operatorname{dim} f(M)=$ 1. The remainder of the argument parallels that of Theorem 1 .

4. Remarks and applications. Theorems 1 and 2 , in many cases, can be interpreted as theorems about zero sets of holomorphic 1-forms. The theorems give, for $D$ with appropriate nonnegativity condition and a holomorphic 1-form pulling back to zero on $D$, a holomorphic mapping $f: M \rightarrow R$ and a holomorphic 1-form $\phi_{R}$ on $R$ with $\phi=f^{*}\left(\phi_{R}\right)$. If the genus $g(R) \geq 2$ then $\phi_{R}$ vanishes at some point $p \in R$. Letting $D_{0}$ be the divisor associated to $f^{-1}(p)$, one has $D_{0} \cdot D_{0}=0$ and $\phi$ vanishes on $D_{0}$. Thus one often expects a divisor $D_{0}$ of nonnegative selfintersection on which $\phi$ actually vanishes (as opposed to pulling back to zero). Even if $g(R)=1$ multiple fibers will give such a $D_{0}$. However, for $g(R)=1$ such a $D_{0}$ does not exist unless there is a fiber all of whose irreducible components are multiple.

Theorems 4 and 5 will show that the genus of an irreducible component of the zero set of a holomorphic 1-form on a compact complex surface $S$ is bounded above by Chern numbers of $S$. We outline this result later. First, defining the genus of a curve $C=\sum_{j=1}^{k} n_{j} C_{j}$ to be $g(C):=\sum_{j=1}^{k} g\left(N C_{j}\right)$ where $g\left(N C_{j}\right)$ is the genus of the normalization of $C_{j}$, we show the following partial converse:

THEOREM 3. Let $S$ be a compact complex surface with the first Betti number $b_{1}(S)$. If $C \subset S$ is any connected curve of nonnegative self-intersection satisfying $g(C) \leq \frac{1}{2} b_{1}(S)-2$ then $C \cdot C=0$ and $C$ is setwise contained in the zero set of some holomorphic 1-form on $S$.

PROOF. Since $g(C) \leq \frac{1}{2} b_{1}(S)-2$ there is at least one holomorphic 1-form on $S$ which pulls back to zero on $C$. To see this, if $b_{1}(S)$ is even then $b_{1}(S)=2 h^{1,0}(S)$ where $h^{1,0}(S)=\operatorname{dim} H^{0}\left(S, \Omega_{S}^{1}\right)$, otherwise $b_{1}(S)=2 h^{1,0}(S)+1$ [5]. In either case $g(C)=\sum_{j=1}^{k} g\left(N C_{j}\right)$ being integral and less than or equal to $\frac{1}{2} b_{1}(S)-2$ implies $\sum_{j=1}^{k} g\left(N C_{j}\right) \leq h^{1,0}(S)-2$. This gives that

$$
\operatorname{dim} \bigcap_{j=1}^{k} \operatorname{ker} i_{j}^{*}=\operatorname{dim} \bigcap_{j=1}^{k} \operatorname{ker} F_{j}^{*} i_{j}^{*} \geq h^{1,0}(S)-\sum_{j=1}^{k} g\left(N C_{j}\right) \geq 2
$$

where $i_{j}: D_{j} \rightarrow S$ is inclusion, $F_{j}: N C_{j} \rightarrow C_{j}$ is normalization, and $\operatorname{ker} i_{j}^{*}=$ $\left\{\phi \in H^{0}\left(S, \Omega_{S}^{1}\right) \mid i_{j}^{*} \phi=0\right\}=\operatorname{ker} F_{j}^{*} i_{j}^{*}$. So $\bigcap_{j=1}^{k} \operatorname{ker} i_{j}^{*}$ nonzero gives a holomorphic 1-form pulling back to zero on $C$. 
Apply Theorem 1 to obtain a holomorphic map $f: S \rightarrow R$ with $C \cdot C=0$ and $C$ setwise contained in a fiber of $f$, say $f^{-1}(p)$. The object is to show $g(R) \geq 2$. Then there is a holomorphic 1 -form $\psi$ on $R$ vanishing at $p$, giving that $f^{*}(\psi)$ vanishes on $f^{-1}(p)$ and therefore on $C$.

In the case $S$ is Kähler, we have, in the notation of $\S 1$,

$$
\begin{aligned}
g(R) & =\operatorname{dim} A(R)=\operatorname{dim} A(S)-\operatorname{dim} \operatorname{span}_{j=1}^{k}\left\langle q_{j}\left(A\left(N C_{j}\right)\right)\right\rangle \\
& \geq \operatorname{dim} H^{0}\left(S, \Omega_{S}^{1}\right)-\sum_{j=1}^{k} \operatorname{dim} q_{j}\left(A\left(N C_{j}\right)\right) \\
& \geq \operatorname{dim} H^{0}\left(S, \Omega_{S}^{1}\right)-\sum_{j=1}^{k} \operatorname{dim} A\left(N C_{j}\right) \\
& =\frac{1}{2} b_{1}(S)-\sum_{j=1}^{k} g\left(N C_{j}\right) \geq 2 .
\end{aligned}
$$

In the case $S$ is non-Kähler, and therefore is elliptic, we have that $h^{1,0}(S)=$ $g(R)+\delta$ where $\delta=0$ or 1 . This yields

$$
\sum_{j=1}^{k} g\left(N C_{j}\right) \leq h^{1,0}(S)-2 \leq g(R)-1
$$

To show $g(R) \geq 2,(4.1)$ allows reduction to the case $\sum_{j=1}^{k} g\left(N C_{j}\right)=0$. Since $C \cdot C=0, \bigcup_{j=1}^{k} C_{j}$ is not an exceptional set in $S$, and so $\bigcup_{j=1}^{k} C_{j}$ is a singular fiber consisting of rational curves. Any elliptic surface containing a singular fiber consisting of rational curves has every holomorphic 1-form as an element of $f^{*} H^{0}\left(R, \Omega_{R}^{1}\right)$ [8]. Hence $h^{1,0}(S)=g(R)$ and (4.1) becomes $0 \leq g(R)-2$.

For completeness, we include the statement of two theorems bounding the genus of an irreducible component of a one-dimensional zero set of a holomorphic 1-form on a compact surface. Full details of the proof appear in [9], but the main idea is sketched here.

THEOREM 4. Let $S$ be a compact complex surface and let $C$ be an irreducible curve contained in the zero set of a holomorphic 1-form $\phi$ on $S$. Then the genus of $C$ is bounded by $g(C) \leq 1+\frac{3}{2} \max \left(0, c_{2}(S)\right)$. by

Sharper bounds on $g(C)$ which depend on the cateogry of the surface $S$ are given

THEOREM 5. Let $S$ be a compact complex surface free from exceptional curves, and let $C$ be an irreducible curve contained in the zero set of a holomorphic 1form $\phi$ on $S$. Let $H_{\phi}=\left\{\psi \in H^{0}\left(S, \Omega_{S}^{1}\right) \mid \psi \wedge \phi \equiv 0\right\}, h_{\phi}=\operatorname{dim} H_{\phi}$, and $h^{1,0}(S)=\operatorname{dim} H^{0}\left(S, \Omega_{S}^{1}\right)$. 
Then $g(C)$, the genus of $C$, is bounded by one of the following:

(1) $g(C)=0$

(2) $g(C) \leq 1$

(3) $g(C) \leq 1+\frac{1}{2} c_{1}^{2}(S)$

(4) $g(C) \leq 1+\frac{c_{2}(S)}{4\left(h_{\phi}-1\right)} \leq 1+\frac{1}{4} c_{2}(S)$

(5) $g(C) \leq 1+\frac{1}{2} c_{2}(S)$ if $S$ is ruled,

if $S$ is elliptic,

if $h^{1,0}(S) \geq 2$ and there exists $\psi \in H^{0}\left(S, \Omega_{S}^{1}\right)$ with $\phi \wedge \psi \not \equiv 0$

if $h^{1,0}(S) \geq 2, S$ is nonruled, and $h_{\phi} \geq 2$ (there exists $\psi \in H^{0}\left(S, \Omega_{S}^{1}\right)$ with $\left.\phi \wedge \psi \equiv 0\right)$ if $h^{1,0}(S)=1$ and $S$ is neither elliptic nor ruled.

Hence, $g(C) \leq 1+\frac{1}{2} \max \left(0, c_{1}^{2}(S), c_{2}(S)\right)$.

The proof of these theorems is based on the fact that $g(C) \leq \pi(C)$, where $\pi(C)=1+\frac{1}{2}(K \cdot C+C \cdot C)$ is the virtual genus of $C$ and $K$ is the canonical divisor of $S$. Theorem 1 gives that $C \cdot C \leq 0$ for $C$ contained in the zero set of a holomorphic 1-form. This gives that $g(C) \leq 1+\frac{1}{2} K \cdot C$. One then bounds $K \cdot C$ or $K \cdot C+C \cdot C$ by containing $C$ in either the canonical divisor $K$ or the fiber of a holomorphic map of $S$ onto a Riemann surface. If $C$ is contained in an effective canonical divisor and $S$ is free from exceptional curves, then $0 \leq K \cdot C \leq K \cdot K$ [5], giving $g(C) \leq 1+\frac{1}{2} K \cdot K$. If $C$ is contained in the fiber of an appropriate holomorphic map, one relies on the fact that $g(C) \leq g(F)$ where $F$ is a regular fiber. The remaining cases to handle are: $S$ is ruled giving $g(C)=0 ; S$ is elliptic giving $g(C) \leq 1 ; S$ maps holomorphically to a Riemann surface $R$ with $g(R) \geq 2$ giving $c_{2}(S) \geq 4(g(F)-1)(g(R)-1)$ and hence $1+c_{2}(S) / 4 \geq g(F) \geq g(C)$; and finally $S$ maps to a one-dimensional Albanese torus with $C$ in a fiber and $K \cdot C \leq c_{2}(S)$. As the above five cases are comprehensive, one easily constructs a universal bound $g(C) \leq 1+\frac{1}{2} \max \left(0, c_{1}^{2}(S), c_{2}(S)\right)$ for $S$ minimal and deduces $g(C) \leq 1+\frac{3}{2} \max \left(0, c_{2}(S)\right)$ for general $S[\mathbf{9}]$.

Finally, in the way of examples of the fiberings of Theorem 1, we remark that in [7] it has been shown that among minimal surfaces of general type there is a class of surfaces admitting holomorphic maps to higher genus Riemann surfaces for which the Chern ratios $c_{1}^{2}(S) / c_{2}(S)$ assume all rational values in the interval $\left[\frac{1}{5}, 3\right]$.

ACKNOWLEDGEMENTS. The work in $\S \S 1$ and 4 is part of the author's Tulane University doctoral thesis written under the direction of Professor Al Vitter. The author would like to thank both Professor Vitter and Professor A. J. Sommese for their advice and guidance.

\section{REFERENCES}

1. E. Bombieri and D. Husemoller, Classification and embeddings of surfaces, Proc. Sympos. Pure Math., vol. 29, Amer. Math., Soc., Providence, R.I., 1975, pp. 329-420.

2. J. B. Carrell and D. I. Lieberman, Holomorphic vector fields and compact Kaehler manifolds, Invent. Math. 21 (1973), 303-309.

3. F. Catanese, On the moduli spaces of surfaces of general type, J. Differential Geom. 19 (1984), 483-515. 
4. H. Grauert, Über Modifikationen und exzeptionelle analytische Menger, Math. Ann. 146 (1962) 331-368.

5. K. Kodaira, On the structure of compact complex surfaces. I, Amer. J. Math. 86 (1964), 751798.

6. C. P. Ramanujam, Remarks on the Kodaira vanishing theorem, J. Indian Math. Soc. 36 (1972), 41-51.

7. A. J. Sommese, On the density of ratios of Chern numbers of algebraic surfaces, Math. Ann. 268 (1984), 207-221.

8. M. J. Spurr, On the zero set of a holomorphic one-form, Thesis, Tulane Univ., 1983.

9. __ On the genus of an irreducible component of the zero set of a holomorphic one-form, Topics in Several Complex Variables, Research Notes in Math., vol. 112, Pitman Press, 1985.

10. K. Ueno, Classification theory of algebraic varieties and compact complex spaces, Lecture Notes in Math., vol. 439, Springer-Verlag, Berlin and New York, 1975.

\section{Department of Mathematics, Rice University, houston, Texas 77251}

Current address: Department of Mathematics, Notre Dame University, Notre Dame, Indiana 46556 